\title{
Musculoskeletal symptoms, skin disorders and visual impairment among fishermen in the Divisional Secretariat Division of Kalpitiya
}

\author{
S R A P Harshani ${ }^{1}$, H T C S Abeysena ${ }^{2}$ \\ (Index words: blindness, fishermen, musculoskeletal disorders, skin disorders, vision)
}

\begin{abstract}
Introduction To describe musculoskeletal symptoms, skin disorders and visual impairment among fishermen in the Divisional Secretariat Division of Kalpitiya.

Methods This was a community based descriptive cross sectional study conducted from August to October 2011. The sample consisted of 465 fishermen with $\geq 6$ months experience in fishing selected using the cluster sampling technique. An interviewer administered questionnaire and a record sheet were used for data collection. Prevalence of the above conditions with $95 \%$ confidence Intervals $(\mathrm{Cl})$ were calculated. Chi square test was applied for the investigation of the factors associated with the above conditions.
\end{abstract}

Results The prevalence of musculoskeletal symptoms was $61 \%(95 \% \mathrm{Cl}: 56.6 \%-65.4 \%)$. The commonest symptom was back pain (37.6\%). The prevalence of skin disorders was $24 \%$ (95\% Cl: 20.1-27.8) and visual symptoms were $38.9 \%$ (95\% Cl: $34.5 \%-43.3 \%)$. The prevalence of visual impairment of either eye was $24.3 \%$ (95\% Cl: $20.3 \%$ $28.3 \%$ ) low vision of either eye $22.4 \%$ (95\% Cl: $18.6 \%$ $26.2 \%)$ and blindness of either eye was $1.9 \%(95 \% \mathrm{Cl}$ : 1.1-2.7). The prevalence of visual impairment of better eye was $16 \%$ (95\% Cl: $12.7-19.3)$, low vision of better eye $15 \%$ (95\% Cl: $12-18.3)$ and blindness of better eye were $0.9 \%$ (95\% Cl: 0.05-1.75). The prevalence of musculoskeletal symptoms, skin disorders or visual impairment did not vary according to service duration in the fishing industry, number of days spent in the boat for a trip or type of boat.

${ }^{1}$ Postgraduate Institute of Medicine, University of Colombo and ${ }^{2}$ Faculty of Medicine, University of Kelaniya, Sri Lanka. Correspondence: HTCSA, e-mail: <chrishantha-abeysena@kln.ac.lk>. Received 2 March and revised version accepted 25 July 2015. 
Conclusions Prevalence of musculoskeletal and skin disorders and visual impairment were relatively higher among the fishermen.

\section{Ceylon Medical Journal 2015; 60: 90-94}

\section{Introduction}

Fishing is one of the major traditional occupations worldwide. Fishing community is unique as they spend their life both in the sea and land. They have their own way of life and cultural values. It is an occupation which pass from generation to generation. No special occupational qualifications or medical examination is necessary to engage in this profession. Accidents and illnesses in the fishing industry are costly. Fishermen have to bear pain and loss of income without any compensation and sometimes even loose their lives during the course of work.

One study reported that standardized incidence ratio for musculoskeletal disorders among fishermen and seamen was significantly higher compared to Danish workforce as a reference population [1]. Another study from USA reported that musculoskeletal symptoms causing work interference in the last 12 months were $38.5 \%$ among fishermen and low back symptoms were the most common cause of work impairment [2]. The main reasons for musculoskeletal symptoms may be the prolonged working hours and lifting heavy weights in incorrect postures. Though the fishing methods have advanced with modern technology, improved facilities in the vessel, motorization and computer technology, musculoskeletal problems are still high among fishermen.

One study reported that the prevalence of the skin diseases including carcinoma was higher among fishermen in North Carolina [3]. Another study compared skin diseases among the fishermen with the out-patient clinic attendees and found a significantly higher percentage among the former population [4]. A study conducted in India found that the prevalence of visual impairment was $30 \%$ and blindness was $7.1 \%$ among the fishing community [5]. Another study in India reported that the prevalence of visual impairment was $54.5 \%$, low vision was $24.6 \%$ and blindness was $7.4 \%$ [6]. The visual defects were nearly five times higher in fishermen than those engaged in nonfishing occupations [6].

In Sri Lanka, the fisheries sector plays a significant role in the economy of the country, contributing to $1.8 \%$ of the gross domestic product and $2.5 \%$ of the total export earnings. This sector provides direct employment to around 2.6 million people [7]. Despite the dangerous nature of fishing, very few studies has been conducted on health of fishermen around the world. There are many differences among fishermen in different countries and within the same country [8]. There are no published data on health problems of fishermen in Sri Lanka. Therefore the objective of this study was to describe musculoskeletal symptoms, skin disorders and visual impairment among fishermen in the Divisional Secretariat Division of Kalpitiya.

\section{Methods}

This was a community-based, descriptive, cross sectional study conducted in the Divisional Secretariat Division of Kalpitiya in Puttalam District from August to October 2011. The study population consisted of 465 fishermen with more than 6 months experience in fishing. An exclusion criterion was fishermen who were living in fishing villages with less than 100 fishing households.

Sample size was calculated assuming a prevalence of visual impairment among fishermen of $50 \%$, precision $5 \%$ at significance level of 5\%. The design effect of 1.2 was considered as the differences of fishermen between fishing villages. The sample size was 465 . Participants were selected using cluster sampling. Fishing villages in the Division with more than 100 fishing householders were taken as clusters and five clusters were selected by simple random sampling. From each cluster 93 eligible fishermen were selected. If there were more than one eligible fisherman in the same household, the most experienced fisherman was selected for the study. If the selected fisherman was not available, the data collection team visited the household following day. A pre-tested interviewer administered questionnaire was administered and a record sheet was used for recording visual acuity. The questionnaire consisted of simple, closed ended questions. Content validity of the questionnaire was assessed. Questionnaire was prepared in English and translated into Sinhala and Tamil by two translators. Retranslation of these two versions again into English was done by another two translators to assess the accuracy of translation. The necessary alterations were made in the Sinhala and Tamil versions accordingly. Two interviewers were trained in data collection at household level. The principal investigator assessed the visual acuity.

Visual acuity was assessed using Snellen's chart. Visual impairment was defined as visual acuity $<6 / 18$ in either eye low vision as visual acuity of $6 / 24$ to $3 / 60$ in either eye and blindness of either eye as visual acuity $<3 / 60$ in either eye. Visual impairment, low vision and blindness of the better eye were defined according to WHO guidelines [9]. Visual impairment was defined as visual acuity of $<6 / 18$ in the better eye. Low vision was defined as visual acuity of $6 / 24$ to $3 / 60$ in the better eye and blindness was defined as visual acuity less than 3/60 in the better eye. Short sightedness was defined as difficulty in seeing long distance and long sightedness as difficulty in seeing near objects. Irritable symptoms of eyes included itching, discharges and redness of the eyes. Musculoskeletal symptoms included pain $>3$ days per week with duration of $>2$ weeks in knee joint or shoulder joint or wrist joint or back. Skin disorders were defined as presence of dermatitis (skin rashes with itching, redness, oozing 
and swelling) or callosities (raised thickened skin over an area of prick injury) or infection (wound with or without a discharge).

Prevalence of musculoskeletal symptoms, skin disorders and visual impairment was calculated with 95\% confidence interval (CI). Chi square test was used for categorised variables. Multiple logistic regression was used where necessary and results were expressed with odds ratio (OR) and 95\% CI. Approval was obtained from the Ethics Review Committee of the Faculty of Medicine, University of Colombo. Informed written consent was obtained from the participants. Those who needed treatment were referred to the relevant clinics.

\section{Results}

A total of 465 fishermen, all males, participated and the response rate was $100 \%$. A majority $(n=371,80 \%)$ had $>10$ years duration of service as a fisherman. Most of

Table 1. Prevalence of musculoskeletal symptoms, skin disorders and visual defects

\begin{tabular}{lcc}
\hline & Prevalence & $95 \%$ CI \\
\hline Musculoskeletal symptoms & $61 \%$ & $56.6-65.4$ \\
Skin disorders & 24 & $20.1-27.9$ \\
Visual symptoms & $39 \%$ & $34.5-43.3$ \\
Visual impairment of either eye & $24.3 \%$ & $20.3-28.3$ \\
Low vision of either eye & $22.4 \%$ & $18.6-26.2$ \\
Blindness of either eye & $1.9 \%$ & $1.1-2.7$ \\
Visual impairment of better eye & $16 \%$ & $12.7-19.3$ \\
Low vision of better eye & $15 \%$ & $11.9-18.3$ \\
Blindness of better eye & $0.9 \%$ & $0.05-1.8$ \\
\hline
\end{tabular}

them $(n=245,52.2 \%)$ worked on single day motor boats and a few $(n=25,5.4 \%)$ in multi day boats. The mean age of the study participants was 38.2 (SD 19) years. One hundred and forty (30\%) were in the age group of 25 to 35 years. Two hundred and seventy two $(88 \%)$ were educated above grade 6 . Two hundred and eighty six $(62 \%)$ were Sinhalese, 372 (80\%) were Catholics and 379 $(85.4 \%)$ were married. Monthly income was less than SLR 10,000 (US \$ 800) per month in $221(47.5 \%)$ participants.

The prevalence of musculoskeletal symptoms was $61 \%$ (Table1). The most common type of musculoskeletal symptom was back pain (37.6\%) followed by knee joint pain $(12.3 \%)$, shoulder joint pain $(7.7 \%)$ and wrist joint pain (3.4\%). Prevalence of the skin disorders was $24 \%$ (Table1). Common type of skin disorders were dermatitis $(10.3 \%)$, callosities $(7 \%)$ and infections $(6.5 \%)$. Skin disorders were commoner in legs (13\%) followed by arms (7\%). Prevalence of visual symptoms was 39\% (Table 1) and $27 \%$ were short sighted, $10 \%$ were long sighted and $0.2 \%$ had tearing and itching of eyes. The prevalence of visual impairment of either eye was $24.5 \%$ low vision of either eye $22.4 \%$ and blindness of either eye were $1.9 \%$. The prevalence of visual impairment of better eye was $16 \%$, low vision of better eye $15 \%$ and blindness of better eye was $0.9 \%$.

The prevalence of musculoskeletal symptoms or skin disorders was not significantly different in those aged $\leq 35$ vs $>35$ years. Time spent in the boat for a trip, single day ( $\leq 1$ day or more) vs other type of the boat and duration of service ( $\leq 10$ years or more). Bivariate analysis showed a statistically significant association between visual impairment of either eye and age and duration of service (Table 2). Multiple logistic regression showed that age $>35$ years had statistically significant association with visual impairment of either eye (Table 3).

Table 2. Factors associated with visual impairment of either eye

\begin{tabular}{|c|c|c|c|c|c|}
\hline & & \multicolumn{2}{|c|}{$\begin{array}{l}\text { Presence of visual } \\
\text { impairment of either eye }\end{array}$} & \multirow[b]{2}{*}{$\chi^{2}$} & \multirow[b]{2}{*}{$p$ value } \\
\hline & & Number & $\%$ & & \\
\hline \multirow[t]{2}{*}{ Age (years) } & $(n=225)$ & 31 & 13.8 & & \\
\hline & $(n=240)$ & 82 & 34.2 & 26.2 & $<0.001$ \\
\hline \multirow[t]{2}{*}{ Duration of boat trip } & $\leq 1$ day $(\mathrm{n}=438)$ & 103 & 23.5 & & \\
\hline & $>1$ day $(\mathrm{n}=27)$ & 10 & 37 & 2.5 & 0.11 \\
\hline \multicolumn{6}{|l|}{ Type of boat } \\
\hline Single day motor boat & $(\mathrm{n}=245)$ & 56 & 22.8 & & \\
\hline Other boats & $(\mathrm{n}=220)$ & 57 & 26 & 0.59 & 0.44 \\
\hline \multicolumn{6}{|l|}{ Service duration } \\
\hline$\leq 10$ years & $(n=94)$ & 15 & 16 & & \\
\hline$>10$ years & $(n=371)$ & 98 & 26.4 & 4.5 & 0.03 \\
\hline
\end{tabular}


Table 3. Factors associated with visual impairment of either eye

\begin{tabular}{lcccc}
\hline Variable & $\beta$ coefficient & $S E$ & OR & 95\% CI \\
\hline $\begin{array}{l}\text { Age }>35 \text { years } \\
\begin{array}{l}\text { Duration of service } \\
>10 \text { years }\end{array}\end{array}$ & 1.15 & 0.25 & 3.2 & $1.9-5.2$ \\
$\begin{array}{l}\text { Number of days spent } \\
\text { in the boat for a trip }\end{array}$ & 0.14 & 0.33 & 1.15 & 0.001 \\
\hline
\end{tabular}

\section{Discussion}

The prevalence of musculoskeletal symptoms among fisherman was $61 \%$ and back pain was the commonest symptom (45.5\%). Prevalence of skin disorders was $24 \%$ and a majority of fishermen had dermatitis $10.3 \%$ followed by callosities (7.1\%) and infections (6.5\%). Prevalence of visual symptoms was $39 \%$ and according to the WHO definition the prevalence of visual impairment was $16 \%$ low vision $15 \%$ and blindness was $0.9 \%$. The prevalence of visual impairment was $24.3 \%$ low vision $22.4 \%$ and blindness of either eye was $1.9 \%$.

A study carried out among fishermen in North Carolina showed that the prevalence of musculoskeletal symptoms causing work interference in the last 12 months was $38.5 \%$ [2]. The difference in prevalence compared to the present study may be due to advanced technology used for fishing in North Carolina resulting in low prevalence of musculoskeletal symptoms. This is the prevalence of symptoms of which interference with work.

Another study done in North Carolina found that the prevalence of eczema was $37 \%$ and fungal infections $30 \%$ [3]. However, the above findings were not based on a representative sample. Another study showed that skin diseases were more common among fishermen compared to an out-patient control group [4]. Skin problems of fishermen may be associated with frequent, prolonged sun exposure, and contact with sea water which results in continuous wetness and contact with marine organisms. Exposure to environmental conditions, especially the extremes of temperature and humidity are also associated with skin lesions. Equipment can cause damage to the skin which can act as an entry point for various infectious organisms [3].

In India the prevalence of visual impairment is $54.5 \%$, low vision is $24.6 \%$ and blindness is $7.4 \%$ among fishermen aged $>50$ years [6]. Another study from India shows that prevalence of visual impairment is $30 \%$ and blindness is $2.7 \%$ [5]. Rate of blindness was similar in both studies. In Sri Lanka the prevalence of visual impairment was 5.9\% and blindness was $1.1 \%$ in the Kandy district among nonfishermen aged $>40$ years [10]. This is less than that reported in our study. Exposure to extreme temperatures, wind and glare cause visual problems [11]. Burke recommends that fishermen protect their eyes with sunglasses [3]. Effective sunglasses block both UVA and
UVB radiation, and polarized glasses block reflected light [9]. We also found that prevalence of visual impairment of either eye were higher among those aged $\geq 35$ years even after controlling for confounding variables.

In summary prevalence of musculoskeletal conditions, skin disorders and visual impairment were relatively higher among fishermen. Service duration in the fishing industry, number of days spent per boat trip and type of boat were not associated with the above conditions. We recommend educating fisherman about prevention of medical conditions and conducting regular medical examination in fishermen.

\section{Conflicts of interest}

There are no conflicts of interest.

\section{References}

1. Kaerlev L, Jensen A, Nielsen PS, et al. Hospital contacts for injuries and musculoskeletal diseases among seamen and fishermen. BMC Musculoskelet Disord 2008; 9: 8 doi:10.1186/1471-2474-9-8.

2. Lipscomb HJ, Loomis D, McDonald MA, et al. Musculoskeletal symptoms among commercial fisheries in North Carolina. Appl Ergon 2004; 35: 417-26.

3. Burke W, Griffith DC, Scott CM, Howell ER. Skin Problems Related to the Occupation of Commercial Fishing in North Carolina. N C Med J 2006; 67: 260-5.

4. Hamdi KI, Al Malikey MA. Frequency of skin diseases among sea fishermen in Basrah. The Int J Dermatol 2009; 7: doi; 10.5580/16f6.

5. Marmaula S, Madala RS, Rao GN. Rapid assessment of visual impairment (RAVI) in marine fishing communities in South India - study protocol and main findings. BMC Ophthalmol 2011; 11(26): doi; 10.1186/ 1471-2415-11-26.

6. Rotti SB, Jainsankar TJ. Distribution of certain diseases among fishermen in Pondicherry: a comparative study. Jawaharlal Institute of Post Graduate Medical Education and Research, Pondicherry Available from https:// www.yumpu.com/en/document/view/8642112/ (accessed on 10th September 2011).

7. Ministry of Fisheries and Aquatic Resources Development, 2013. Available from http://www.fisheries.gov.lk/ content.php?cnid=ststc

8. Lawrie. T, Matheson C, Ritchie L, et al. The health and life 
style of Scottish fishermen: a need for health promotion. Health Educ Res 2004; 19: 373-9.

9. World Health Organisation. International Classification of Disease and Related Health Problems $10^{\text {th }}$ Version, World Health Organization, Geneva, 2007.

10. Edussuriya K, Senanayake S, Senaratne T, et al. The prevalence and causes of visual impairment in central Sri Lanka. Opthalmol 2009; 116: 52-6.

11. European Agency for Safety and Health at Work. Help for Small and Medium Enterprises-Fisheries Sector. Available from http:/osha.europa.eu/en/sub/sme/products/fisheries/ index.htm (accessed on $10^{\text {th }}$ September 2011). 